\title{
Quality Assessment of GOCE Gradients
}

\author{
Brieden P. (1), Müller J. (1, 2)
}

(1) Institut für Erdmessung, Leibniz Universität Hannover, Schneiderberg 50, 30167 Hannover, Germany

(2) Centre for Quantum Engineering and Space-Time Research (QUEST), Welfengarten 1, 30167 Hannover, Germany

\begin{abstract}
The first ESA Earth Explorer Core Mission GOCE (Gravity Field and Steady-State Ocean Circulation Explorer) entered the operational measurement phase in September 2009. Before gravity field processing, the quality of the GOCE gradients in the measurement bandwidth $(5-100 \mathrm{mHz}), \mathrm{MBW}$, has to be assessed. Here, two procedures have been developed in Hanover, the mutual comparison and analysis of observed gradients in satellite track crossovers and the application of terrestrial gravity data which are upward continued and transformed into reference gradients for the GOCE gradiometer measurements.

First the gravity gradients are filtered, where the longer wavelength signals below the MBW are replaced by global geopotential model (GPM) information. The filtered time series is used as input signal for both validation methods. ${ }^{1}$
\end{abstract}

\section{Pre-Processing}

The GOCE gravity gradients result from a combination of differential mode accelerations along and inertial angular rates about the three gradiometer axis on board the GOCE satellite. Because of accelerometer drift as a natural and expected behavior of the accelerometers, gravity gradients are affected by drift, too. This leads to inaccuracies in the gravity gradients over long periods.

The GOCE gradiometer has been designed such that the error characteristics of the gravity gradients are optimized in the so-called measurement bandwidth (MBW). The MBW of the GOCE gradiometer is between 5 and 100 millihertz $(\mathrm{mHz})$. It is the spectral domain where validation is primarily required.

\subsection{The idea}

For both validation methods, which are presented in this paper, full signal information is needed, so a simple high-pass filtering of the time series is not sufficient. The goal is to get the signal with the highest possible quality (for validation of the gradiometer data), both at low frequencies (for reduction purposes only) and in the MBW. The idea is to combine shortwavelength signal information from GOCE measurements with long wavelength signal input on the basis of a global geopotential model (GPM). The resulting, combined signal is used as input for the validation methods.

\subsection{Filtering}

In the analysis, a FIR (finite impulse response) filter with a cut-off frequency of $5 \mathrm{mHz}$ is used to high pass filter the time series of the GOCE gradients and low pass filter a gradients' time series computed on the basis of a GPM in GOCE altitude and attitude. Filtering is performed separately for the time series of all tensor components. The GPM that is used in the following analysis is the ITG-Grace2010s [5] up to degree and order 180.

\footnotetext{
1 REMARK

A similar paper has been presented at the ESA Living Planet Symposium under the title »TWO METHODS FOR QUALITY ASSESSMENT OF GOCE GRADIENTS « [1]. It obtained the publication number GEOTECH-1396 of the German Ministry of Education and Research (BMBF) R\&D-Programme GEOTECHNOLOGIEN. The present paper has been modified and data of another period (November 2009) was investigated.
} 


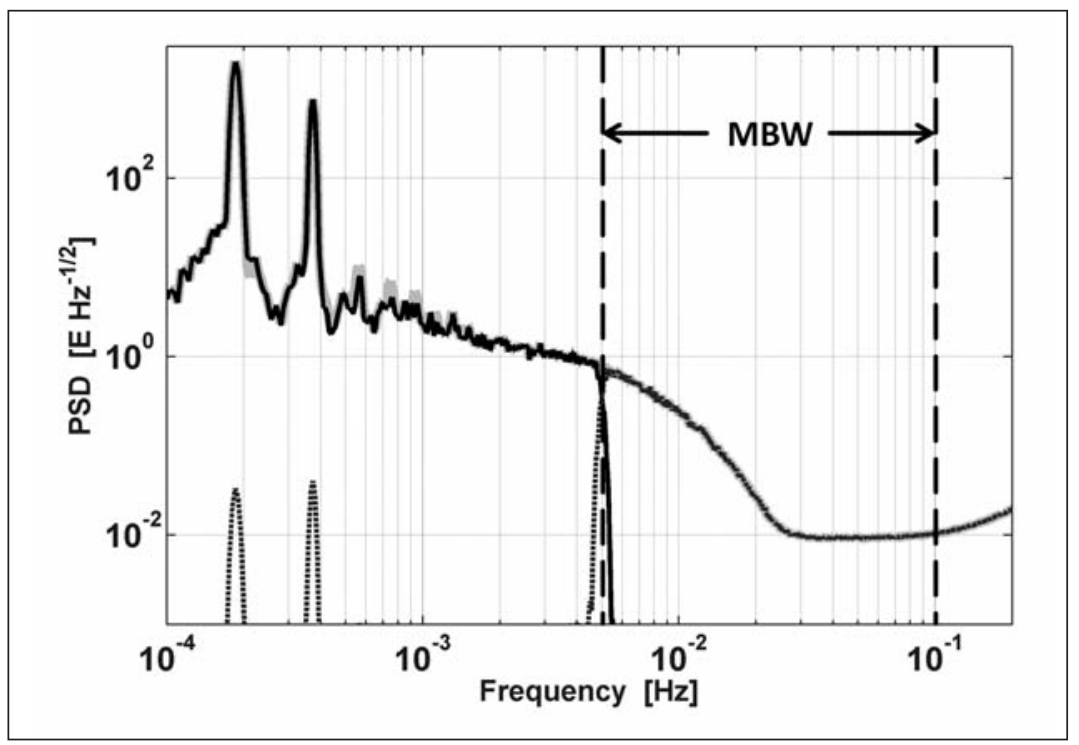

Power Spectral Density (PSD) of the GOCE gravity gradient tensor component $V_{y y}$ (gray), its high pass filtered part (dotted gray) and the low pass filtered part of the ITG-Grace2010s (black)

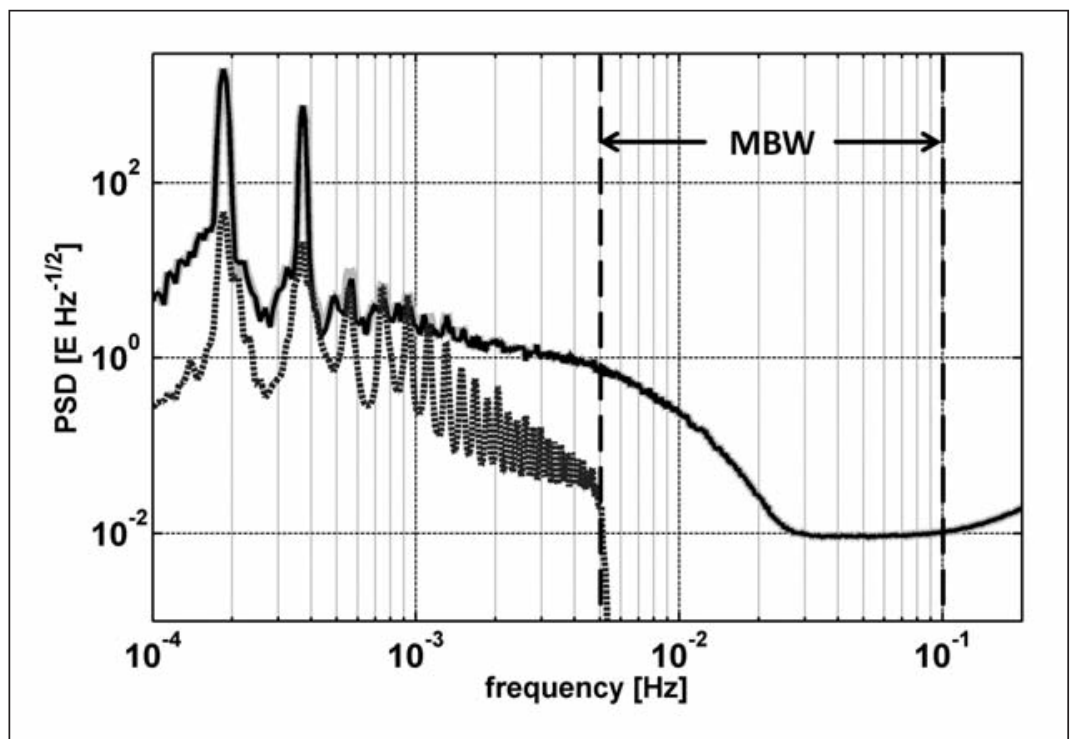

Figure 2: Power spectral density of the original GOCE gradient $V_{y y}$ time series (gray), the filter result (black) and the differences (dotted gray)

Figure 1 shows the power spectral density (PSD) of the GOCE gravity gradient component Vyy in gray, its high pass filtered part (dotted gray) and the low pass filtered part of the ITGGrace2010s in black.

In order to achieve the best possible quality assessment, the resulting combined filtered GOCE signal has also been low pass filtered to remove possible errors above $50 \mathrm{mHz}$. This upper cutoff frequency corresponds to a spherical harmonic degree of about 270, where no significant gravity signal is expected as there the signal level is below the noise level.

The resulting gradient PSD after filtering is illu- strated in Figure 2, where the PSD of the GOCE measurements (gray), the filtered signal (black) and the differences between these two (dotted gray) are shown. The differences in the MBW are very small, about four orders of magnitude below the signal power. It is obvious that GOCE also has good signal quality below the MBW down to $1.5 \mathrm{mHz}$.

The resulting time series of the gravity gradients, consisting of long-wavelength information from ITG-Grace2010s and short-wavelength information from GOCE measurements (black PSD in Figure 2), is used as input for our validation analysis. The data period is one month: November 2009. 


\section{Reference Gradient Approach}

The idea of the reference gradient approach is based on regional gravity field data over well surveyed areas, that is upward continued to gradients at satellite altitude, serving as reference gradients for the GOCE data. These reference gradients in the GOCE observation frame can then be used for evaluating the quality of the measured gradients.

\subsection{The reference gradient method}

The reference gradient approach is based on regional gravity data in terms of global geopotential models (GPM), terrestrial gravity anomalies and residual terrain models (RTM). The terrestrial data $\left(\Delta \mathrm{g}_{\mathrm{G}}\right)$, a combination of terrestrial gravity anomalies, airborne gravimetry and satellite altimetry, is reduced from long-wavelength $\left(\Delta g_{M}\right)$ and short-wavelength $\left(\Delta g_{R T M}\right)$ contributions (remove-restore-technique):

$\Delta g^{r}=\Delta g_{G}{ }^{-} \Delta g_{M}{ }^{-} \Delta g_{R T M}$.

The resulting residual gravity anomalies $\Delta \mathrm{g}^{r}$ (Eq. 1) are upward continued into GOCE altitude, transformed to residual 'disturbing gradients' $T_{i j}$ and restored to full tensor components $V_{i j}$ ref, as it is shown in Figure 3. These computed, so called, reference gradients $V_{i j}$ ref and the GOCE gradiometer measurements $V_{i j}{ }^{G}$ are compared; the differences $\Delta \mathrm{V}_{\mathrm{ij}}^{\mathrm{RG}}$ are further analyzed.

A detailed explanation of the procedure of reference gradient determination and the reference gradient approach can be found in [2], [6] and [7].

\subsection{Analysis of the gradient differences}

The current regional evaluation area is central Europe. Hence, only satellite tracks that cross this area and are at least 200 seconds long are evaluated. The differences of each tensor component are reduced by a mean value. Ideally the mean value is zero; in practice, offsets remain indicating a constant difference between the filtered GOCE gradients and the computed reference gradients. The remaining constant differences might be caused by the filtering of the data or some inconsistent choice of datum parameters. Future work will focus on possible reasons for those constant differences.

\subsection{Reference gradient results}

Figure 4 shows the mean-reduced gray-coded differences $\Delta \mathrm{V}_{\mathrm{yy}}$ within the evaluation area. In the given data set, a mean value of $-132 \mathrm{mE}$ has been reduced. The differences in $\Delta \mathrm{V}_{x x}$ and $\Delta \mathrm{V}_{\mathrm{yy}}$ have a magnitude of about 11 to $15 \mathrm{mE}$. The differences in $\Delta \mathrm{V}_{z z}$ are slightly worse with a magnitude of about $25 \mathrm{mE}$.

The residual differences are to be analysed along each track (Figure 4). The analysis is carried out in the frequency domain to identify relevant frequencies in the differences. Figure 5 shows the power spectral densities (PSD) of the differences along each track within the evaluation area. Each of the black, grey and white curves specifies the PSD of one track. The average of all PSDs is given by the thick gray line. The requirements of the GOCE tensor trace is pictured in dotted black. Obviously, almost all results meet the requirements. Some of the PSD curves show single spectral signals

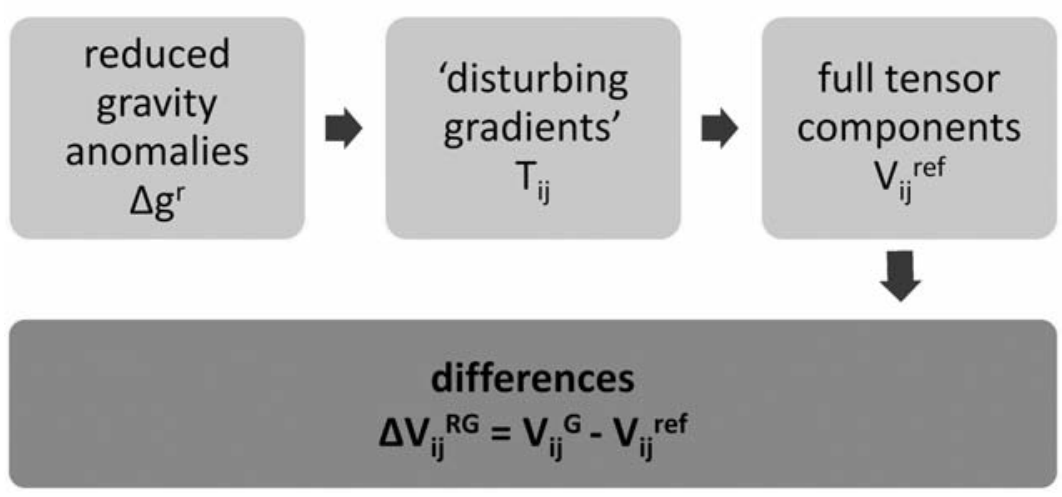

Figure 3: Computation steps from terrestrial gravity data to gradient differences of GOCE measurements 


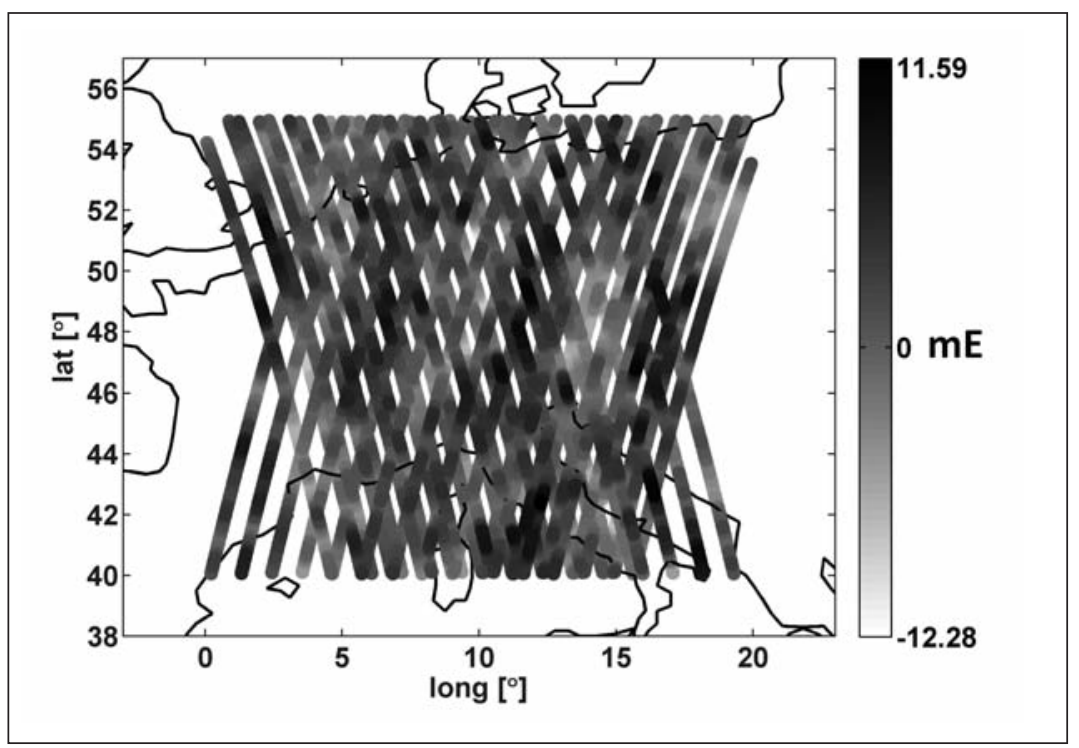

Figure 4: Residual differences $\Delta \mathrm{V}_{\mathrm{yy}}[\mathrm{mE}]$, mean value of $-132 \mathrm{mE}$ is reduced

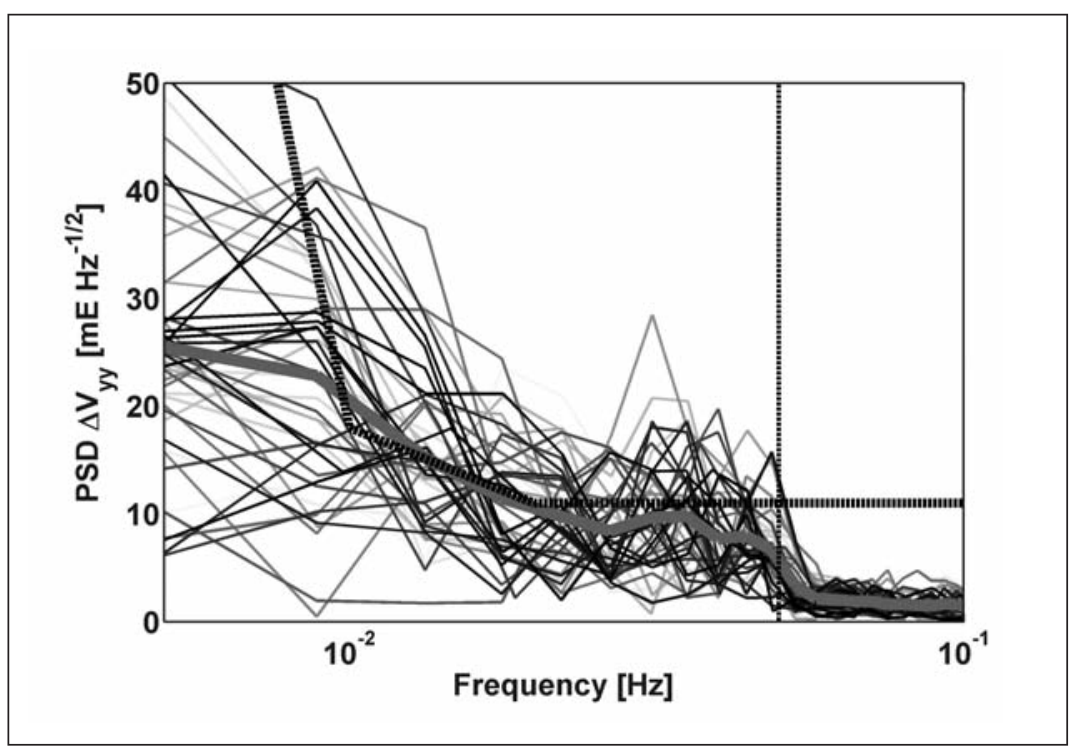

Figure 5: PSD of each track within the evaluation area (black to white), the averaged PSD (wide gray line) and the requirement of the GOCE trace (dotted black)

above the requirements, but one has to have in mind, that each PSD is computed on the basis of very short time-series of only $200 \mathrm{~s}$. The effect of the second filtering at $50 \mathrm{mHz}$ that is described in chapter 1.2 is also visible in the PSD of the differences in Figure 5. The cut-off frequency of $50 \mathrm{mHz}$ is indicated by the vertical dotted black line.

It is a very good result having achieved a noise performance of 10 (higher frequencies) to $25 \mathrm{mE} / \sqrt{ } \mathrm{Hz}$ (lower frequencies) for $\mathrm{V}_{\mathrm{yy}}$. The performance of $V_{z z}$ is slightly larger, up to about $35 \mathrm{mE} / \sqrt{ } \mathrm{Hz}$.

\section{Cross-Over Approach}

The cross-over (XO) approach is a relative validation method, i.e., a procedure using GOCE data only. XO analysis allows checking the gravity gradients at the accuracy level expected for GOCE.

\subsection{The XO method}

The idea of the XO approach is to use the geographical correlation between two GOCE tracks crossing each other over the same point on earth, in which the gradiometer should measure the same gravity gradients. 


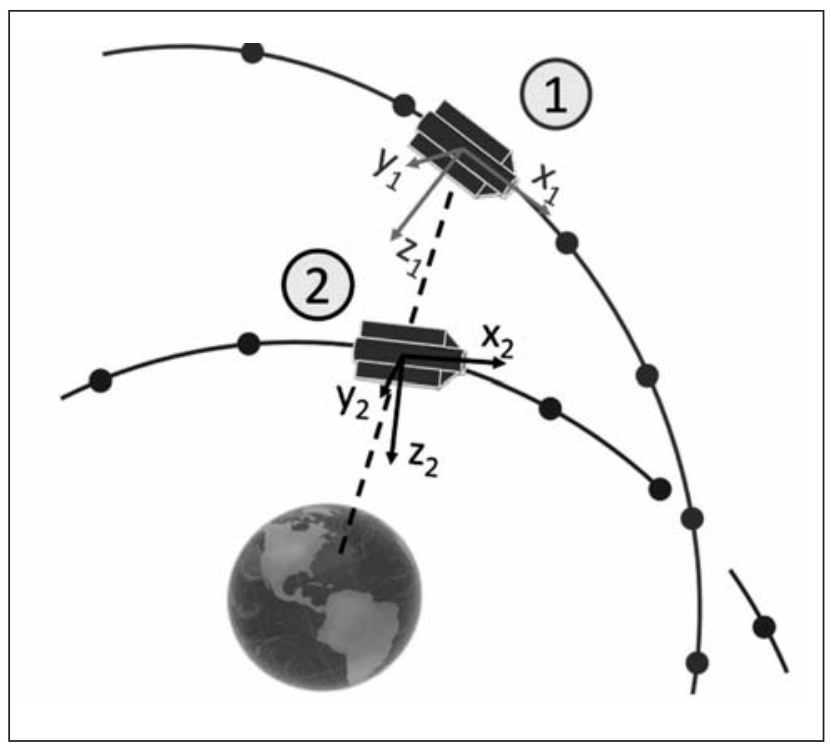

Figure 6: Principle of the $X O$ approach - different attitude and altitude of the GOCE gradiometer in two orbits crossing each other over the same point on earth

What has to be considered are differences in attitude and altitude between the two satellite and gradiometer positions (Figure 6). These differences have to be reduced for the XO analysis. The reduction of attitude and altitude effects is performed by using the global geopotential model ITG-Grace2010s [5]. Reference values for the measured gradients in the $\mathrm{XO}$ are computed in the gradiometer reference frame (GRF) for both gradiometer positions. The resulting accuracy of those gradients is obviously not high enough, since the GPM is less accurate in the MBW than the GOCE measurements. The idea of the $\mathrm{XO}$ approach is that the difference of reference gradients in the two gradiometer positions is the same as the difference of real GOCE measurements. Based on this assumption, the differences between the real measurements are reduced by using the differences between the reference values:

$$
\Delta V_{i j}^{X O}=V_{i j, G}^{1}-V_{i j, G}^{2}-\left(V_{i j, G P M}^{1}-V_{i j, G P M}^{2}\right) \text {. }
$$

The residual gradient differences $\Delta \mathrm{V}_{\mathrm{ij}} \mathrm{xo}$ that should be zero in the ideal case are then further analyzed, where $\mathrm{V}_{\mathrm{ij}, \mathrm{G}^{1}}$ and $\mathrm{V}_{\mathrm{ij}, \mathrm{G}^{2}}$ are the measured GOCE gradients in the two satellite positions (see Figure 6) and $V_{i i, G P M}{ }^{1,2}$ are the corresponding tensor components that are computed from the ITG-Grace2010s.

A detailed description of the procedure of cross-over analysis and its application for validating GOCE gravity gradients is given in [2], [3], [4] and [6].

\section{2. $X O$ results}

Based on about one month of GOCE data from November 2009, the XO approach produces a set of residual differences $\Delta \mathrm{V}_{\mathrm{ij}} \mathrm{xo}$ according to Eq. (2). Even an ideal reduction of the GPM part provided, the differences are not always reduced to zero because GOCE data might contain some anomalies that are to be detected with this validation approach. To get a first impression, a threshold of maximum allowed difference in the $\mathrm{XO}$ is selected. The statistics in Table 1 points out, how much percentage of the differences in the cross-over points are below the selected threshold and thus are good XOs. Table 1 gives an overview for the main diagonal components of the gravity gradient tensor.

Obviously for a given threshold of only $11 \mathrm{mE}$, more than $97 \%$ of the differences in all cross over points are below this limit for the $X X$ and $Y Y$ tensor component, which is a very good result. Despite a higher threshold, the ZZ component shows a slightly less accurate behaviour. Taking a threshold of $20 \mathrm{mE}$ for ZZ, the percentage of differences exceeding it is reduced to about $1 \%$. 
Table 1: Percentage of good XOs that are below a given threshold depending on the tensor component

\begin{tabular}{|ccc|}
\hline $\begin{array}{c}\text { tensor } \\
\text { component }\end{array}$ & $\begin{array}{c}\text { threshold } \\
\text { [mE] }\end{array}$ & $\begin{array}{c}\text { percentage of } \\
\text { good XOs }\end{array}$ \\
\hline$X X$ & 11 & $99.3 \%$ \\
\hline$Y Y$ & 11 & $97.5 \%$ \\
\hline$Z Z$ & 15 & $94.8 \%$ \\
\hline
\end{tabular}

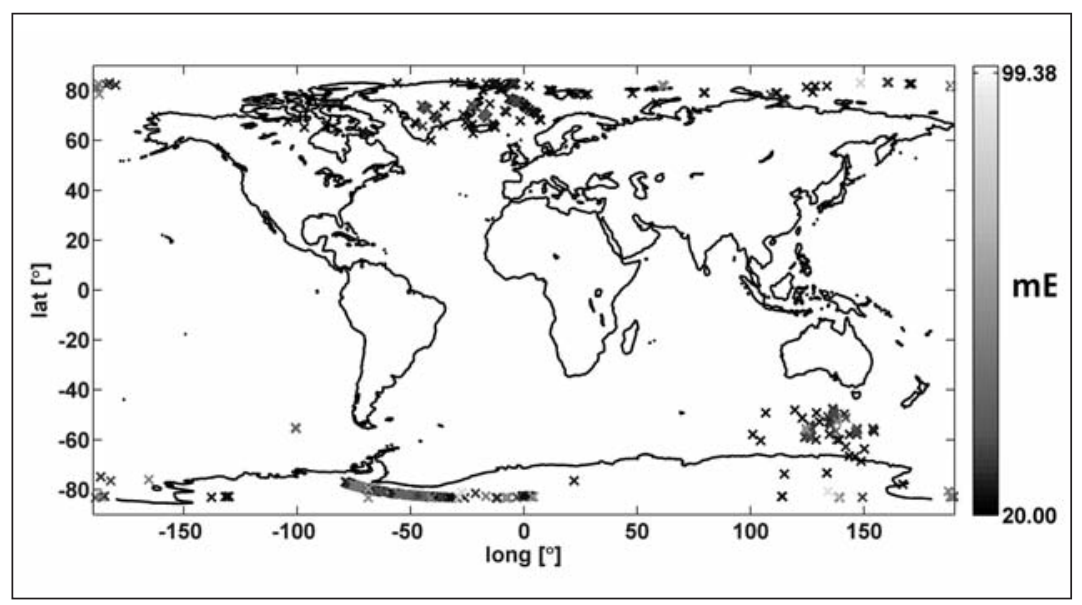

Figure 7: Gray-coded XOdifferences $[\mathrm{mE}]$ of tensor component $V_{y y}$ that exceed a threshold of $20 \mathrm{mE}$, data base: November 2009

For gravity field processing the highly accurate main diagonal components of the gravity gradient tensor are used. The $\mathrm{XO}$ results show that more than $97 \%\left(V_{x x}\right.$ and $\left.V_{y y}\right)$ of the main diagonal components achieve the expected quality level.

The differences of tensor component $V_{y y}$ that exceed a threshold of $20 \mathrm{mE}$ are shown in Figure 7. It gives the geographical distribution of the gray-coded differences that almost exclusively can be found near the poles. That is not astonishing, as most of the XO's occur in high latitudes. The XO approach also allows to identify individual inaccurate measurements along orbit parts. Moreover, accumulations of differences in certain areas like south of Australia are detected.

As Figure 7 also shows, almost all the threshold-exceeding differences have values close to the threshold and only very few of them have bigger amplitudes. Nevertheless some scattered larger differences appear which will be subject to future investigations.

The results reflect the good suitability of the XO approach to validate the GOCE data at the level of some $\mathrm{mE}$. The $\mathrm{XO}$ approach underlines the good data quality of the GOCE gravity gradients.

\section{Conclusions and Outlook}

The inaccuracies in the longer wavelengths in the GOCE gravity gradients are improved with long wavelength information from the ITGGrace2010s. The GOCE gravity gradients are filtered first. Here, a finite impulse response filter (FIR) is applied. In the future, the determination of a best cut-off frequency will be investigated. The resulting filtered time series is used as input for both Hanover validation methods.

On the basis of about one month (Nov. 2009) 
of GOCE data, the results of the reference gradient approach show residual differences between the measured and the reference gradients at about 10 to $15 \mathrm{mE}$ for the $X X$ and $Y Y$ tensor components. The differences for the $Z Z$ component are slightly larger up to about 25 $\mathrm{mE}$. The differences in the spectral domain show a noise performance up to about 25 $\mathrm{mE} / \mathrm{JHz}(X X, Y Y)$ to $35 \mathrm{mE} / \mathrm{JHz}$ (ZZ) for the main diagonal components in the measurement bandwidth.

The results of the cross-over (XO) approach give a similar picture: more than $99 \%$ of the residual differences in the cross-over points are smaller than $15 \mathrm{mE}$ for the $\mathrm{XX}$ and $\mathrm{YY}$ components. The percentage of differences in $Z Z$ exceeding $15 \mathrm{mE}$ is $95 \%$, but ZZ is also at the level of $99 \%$ for a threshold of $20 \mathrm{mE}$.

In summary, on the basis of about one month of GOCE data, both approaches have proved their suitability to validate GOCE data. The noise performance of the tensor component $V_{z z}$ is slightly worse compared to the other main diagonal components. The two validation methods underline the very good quality of the GOCE gravity gradients.

\section{Acknowledgement}

The project REAL GOCE is part of the R\&D Programme GEOTECHNOLOGIEN. GEOTECHNOLOGIEN is funded by the German Ministry of Education and Research (BMBF) and the German Research Foundation (DFG), Grant (03G0726C) of project REAL GOCE.

\section{References}

[1] Brieden, P., Müller, J. (2010): Two Methods for Quality Assessment of GOCE Gradients, ESA Living Planet Symposium, Conference proceedings, ESA SP-686, Bergen (Norway), 2010.

[2] Jarecki, F., Wolf, K.I., Denker, H., Müller, J. (2006): Quality assessment of GOCE gradients. In: Flury, J. et al. (eds.), Observation of the Earth System from Space, Springer, New York, pp. 271-285.
[3] Jarecki, F. (2010): Kreuzungspunktdifferenzen zur relativen Validierung satellitengradiometrischer Messungen, Wissenschaftliche Arbeiten der Fachrichtung Geodäsie und Geoinformatik der Leibniz Universität Hannover, ISSN 0174-1454, Nr. 286, 2010.

[4] Jarecki, F., Müller, J. (2009): Robust trend estimates from GOCE SGG satellite track crossover differences. In: Sideris, M. (ed.), Observing Our Changing Earth. IAG Symposia 133, Springer, New York, pp. 363-370.

[5] Mayer-Gürr, T., Kurtenbach, E., Eicker, A. (2010): The static solution ITG-Grace2010s. Available at: http://www.igg.uni-bonn.de/apmg/ index. php?id=itg-grace 2010 .

[6] Müller, J., Jarecki, F., Wolf, K.I., Brieden, P. (2010): Quality Evaluation of GOCE Gradients. In: Flechtner, F.M., (eds.), System Earth via Geodetic-Geophysical Space Techniques, Springer, New York, pp. 265-276.

[7] Wolf, K.I. (2007): Kombination globaler Potentialmodelle mit terrestrischen Schweredaten für die Bestimmung der zweiten Ableitungen des Gravitationspotentials in Satellitenbahnhöhe, Ph.D. thesis, Deutsche Geodätische Kommision, Reihe C Nr. 603, www.dgk.badw. de, identical with: Wissenschaftliche Arbeiten der Fachrichtung Geodäsie und Geoinformatik der Leibniz Universität Hannover, ISSN 01741454, Nr. 264, Hannover 2007. 